\title{
The B7H4-PDL1 classifier stratifies immuno-phenotype in cervical cancer
}

\author{
Lingyan Chen ${ }^{1 \dagger}$, Jianfeng Dong ${ }^{2 \dagger}$, Zeying $\mathrm{Li}^{3 \dagger}$, Yu Chen ${ }^{1 *}$ and Yan Zhang ${ }^{1 *} \mathbb{C}$
}

\begin{abstract}
Background: It has been revealed that B7H4 is negatively correlated with PDL1 and identifies immuno-cold tumors in glioma. However, the application of the B7H4-PDL1 classifier in cancers has not been well testified.

Methods: A pan-cancer analysis was conducted to evaluate the immunological role of B7H4 using the RNAsequencing data downloaded from the Cancer Genome Atlas (TCGA). Immunohistochemistry (IHC) and multiplexed quantitative immunofluorescence (QIF) were performed to validate the primary results revealed by bioinformatics analysis.

Results: The pan-cancer analysis revealed that B7H4 was negatively correlated with PDL1 expression and immune cell infiltration in CeCa. In addition, patients with high B7H4 exhibited the shortest overall survival (OS) and relapsefree survival (RFS) while those with high PDL1 exhibited a better prognosis. Multiplexed QIF showed that B7H4 was mutually exclusive with PDL1 expression and the B7H4-high group exhibited the lowest CD8 + T cell infiltration. Besides, B7H4-high predicted highly proliferative subtypes, which expressed the highest Ki67 antigen. Moreover, B7H4-high also indicated a lower response to multiple therapies.
\end{abstract}

Conclusions: Totally, the B7H4-PDL1 classifier identifies the immunogenicity and predicts proliferative subtypes and limited therapeutic options in $\mathrm{CeCa}$, which may be a convenient and feasible biomarker in clinical practice.

Keywords: B7H4, PDL1, Tumor immunity, Pan-cancer

\section{Background}

With the development of treatment strategies for malignant cancers, immune checkpoint blockade (ICB) is emerging as inspiring immunotherapy transforming the standard of treatment [1]. Representative immune checkpoint inhibitors are monoclonal antibodies that interfere with the interaction between programmed cell death 1 (PD1) and programmed death-ligand 1 (PDL1) inhibitory proteins expressed on the surface of $\mathrm{T}$ cells and tumor cells, respectively [2, 3]. High infiltrating levels of effector

\footnotetext{
*Correspondence: cyidiom1981@yeah.net; zhangyan2020@njmu.edu.cn †Lingyan Chen, Jianfeng Dong and Zeying Li contributed equally to this work

1 Department of Oncology, Wuxi Maternal and Child Health Hospital

Affiliated to Nanjing Medical University, No. 48 Huaishu Road, Wuxi 214000, China

Full list of author information is available at the end of the article
}

immune cells are essential for the response to immunotherapy. Typically, tumors with a high effector immune cells infiltration are defined as "hot" tumors, in which PDL1 is adaptively upregulated [4]. In clinical practice, the detection of PDL1 is considered a predictive biomarker for anti-PD1/PDL1 therapies [5]. Patients with high PDL1 expression tended to be highly responsive to PD1/PDL1 blockade [6].

In addition to PDL1, B7H4 is highly expressed in tumor tissues, making them attractive candidate immunotherapeutic targets and biomarkers [7, 8]. B7H4 was first discovered in 2003 due to its sequence similarities with other B7 family members $[9,10]$. B7H4 encodes a heavily glycosylated membrane protein, and negatively regulates $\mathrm{T}$ cell activation by limiting proliferation, cytokine production, and cytotoxicity $[9,10]$. According to previous research, co-expression of PDL1 and B7H4 is rare in original author(s) and the source, provide a link to the Creative Commons licence, and indicate if changes were made. The images or other third party material in this article are included in the article's Creative Commons licence, unless indicated otherwise in a credit line to the material. If material is not included in the article's Creative Commons licence and your intended use is not permitted by statutory regulation or exceeds the permitted use, you will need to obtain permission directly from the copyright holder. To view a copy of this licence, visit http://creativecommons.org/licenses/by/4.0/. The Creative Commons Public Domain Dedication waiver (http://creativeco mmons.org/publicdomain/zero/1.0/) applies to the data made available in this article, unless otherwise stated in a credit line to the data. 
several cancers, including lung cancer and breast cancer $[11,12]$. Moreover, B7H4 is negatively correlated with PDL1 and identifies immuno-cold tumors in glioma [13]. However, as we all know, the blood-brain barrier blocks the entry of the immune cells and therapeutic drugs to tumor tissues and brain parenchyma to a great extent [14]. Thus, the application of the B7H4-PDL1 classifier in glioma might not be a good option.

In the current research, we conducted a pan-cancer analysis of the correlation between $\mathrm{B} 7 \mathrm{H} 4$ and immunological features. We found that $\mathrm{B} 7 \mathrm{H} 4$ was negatively correlated with immunological features in several cancers, including cervical cancer ( $\mathrm{CeCa}$ ), glioma and melanoma. We selected $\mathrm{CeCa}$ for further analysis and found that B7H4 was negatively correlated with PDL1 and immune cells infiltration. Briefly, B7H4 high expression identifies immuno-cold subtype in $\mathrm{CeCa}$. It was also suggested that $\mathrm{B} 7 \mathrm{H} 4$ predicted the hyperproliferative subtype in $\mathrm{CeCa}$. Overall, the application of the B7H4-PDL1 classifier in CeCa may be a better choice.

\section{Methods}

Public data acquisition and Bioinformatics analysis

The pan-cancer normalized RNA-sequencing (RNA-seq) datasets and corresponding clinical information from the Cancer Genome Atlas (TCGA) dataset were downloaded from Xena (https://xenabrowser.net/datapages/). The abbreviations for various cancer types were given in Additional file 1: Table S1. Besides, the data of RNA-seq and clinical information in the Chinese Glioma Genome Atlas (CGGA) database (mRNA-array_301) were also obtained from the official website (http://www.cgga.org. cn/index.jsp).

The bioinformatics analysis in this research contained public data acquisition, pan-cancer analysis of the correlation between $\mathrm{B} 7 \mathrm{H} 4$ and immunological features, evaluation of the immunological features of the TME in $\mathrm{CeCa}$, calculation of the enrichment scores of immunotherapy-related gene signatures and prediction of therapeutic response. The detailed description of bioinformatics analysis was exhibited in Additional file 1: Additional Methods.

\section{Clinical samples}

The CeCa tissue microarray (TMA, Cat. HUteS168Su01) was obtained from Outdo BioTech (Shanghai, China). The TMA contained $126 \mathrm{CeCa}$ tissues and 42 paired adjacent tissues. Detailed clinico-pathological characteristics follow-up data of the cohorts were provided by Outdo BioTech as well and shown in original data. Besides, we also $30 \mathrm{CeCa}$ tissues submitted for multiplexed quantitative immunofluorescence (QIF) in this research and all the patients were recruited by the Wuxi Maternal and Child Health Hospital Affiliated to Nanjing Medical University. Ethical approval for the use of the TMA was granted by the Clinical Research Ethics Committee in Wuxi Maternal and Child Health Hospital Affiliated to Nanjing Medical University.

\section{IHC and semi-quantitative evaluation}

Immunohistochemistry (IHC) staining was conducted on the TMA. The primary antibodies used were as follows: anti-B7H4 (1:100 dilution, Cat. ab252438, Abcam, Cambridge, UK), anti-PD-L1 (Ready-to-use, Cat. GT2280, GeneTech, Shanghai, China), anti-KI67 (Ready-to-use, Cat. GT2101, GeneTech, Shanghai, China) and antiEGFR (Ready-to-use, Cat. PA192, Abcarta, Suzhou, China). Antibody staining was visualized with $\mathrm{DAB}$ and hematoxylin counterstain, and stained sections were scanned using Aperio Digital Pathology Slide Scanners. A total of 118 TMA points were retained for further analysis after the exfoliated points were removed. All stained sections were independently evaluated by two independent pathologists using the immunoreactivity score (IRS) criterion [15].

\section{Definition of the positive rates of $\mathrm{B} 7 \mathrm{H} 4$ and PDL1 in CeCa}

To identify the immune subtypes in $\mathrm{CeCa}$ based on the B7H4-PDL1 classifier, we first defined the positive staining with the threshold of IRS $\geq 3$ in the IHC cohort. Thus, PDL1 was $36.44 \%$ positive in the cohort and B7H4 was $19.49 \%$. For the patients in the TCGA dataset, we defined PDL1 as positive expression with the threshold of top $35 \%$ and $\mathrm{B} 7 \mathrm{H} 4$ as positive expression with the threshold of top $20 \%$ by referring to positive rate in the IHC cohort.

\section{Multiplexed quantitative immunofluorescence}

To measure the levels of PD-L1, B7-H4 and CD8 in the $\mathrm{CeCa}$ samples, the multiplexed QIF was directly performed on the tissue section using a previously described protocol with simultaneous detection of DAPI [16]. The primary antibodies were as follows: anti-B7H4 (1:100 dilution, Cat. ab252438, Abcam, Cambridge, UK), antiPDL1 (1:500 dilution, Cat. ab237726, Abcam, Cambridge, UK) and anti-CD8 (1:200 dilution, Cat. ab101500, Abcam, Cambridge, UK). The expression levels of B7H4 and PDL1 were evaluated according to the previous method. For CD8 staining, infiltration level was assessed by estimating the percentage of cells with strong intensity of membrane staining in the stroma cells. For stratification, the B7H4 and PDL1 levels were classified as high/ low using the top 50-percentile of the cohort scores as stratification cut-point. 


\section{Statistical analysis}

All statistical analyses were applied by $\mathrm{R}$ version 4.0.0. Wilcoxon rank-sum test was used to measure the difference between groups with continuous values, while Fisher exact method for evaluating the difference among grouping variables. For all analyses, a two-paired $\mathrm{P}$-value $<0.05$ was regarded as statistical significance if not noted.

\section{Results}

The prognostic value of the B7H4-PDL1 classifier in glioma According to the report of Chen et al, the positive rates of $\mathrm{B} 7 \mathrm{H} 4$ and PDL1 were approximately $20 \%$ revealed by IHC analysis [13]. We defined the positive $\mathrm{B} 7 \mathrm{H} 4$ and PDL1 expression as the top $20 \%$ expression, and then divide glioma patients into three major subgroups in both TCGA and CGGA datasets. Similarly, the co-high expression of B7H4 and PDL1 was rare (Fig. 1A, B). Besides, Chen et al. declared that the B7H4-PDL1 classifier distinguished different immunogenicity in glioma [13]. Based on molecular characteristics, B7H4-high tumors could be defined as "cold" tumors, PDL1-high tumors were "hot" tumors, and co-low tumors were somewhere in between. Generally, patients with "hot" tumors exhibited a better prognosis and higher therapeutic response [17-19].
However, this assumption was not supported by survival analysis. As shown in Additional file 2: Figure S1, patients with high PDL1 expression exhibited the shortest overall survival (OS) (Fig. 1C, D). What's more, in the CGGA dataset, patients with high $\mathrm{B} 7 \mathrm{H} 4$ expression exhibited the longest OS, but whose gliomas should be defined as "cold" tumors (Fig. 1D). Overall, these data uncover the B7H4-PDL1 classifier maybe not a better choice in glioma, especially among the Chinese population.

\section{Pan-cancer immunological correlation of $\mathrm{B} 7 \mathrm{H} 4$}

To explore the appropriate cancer types in which the B7H4-PDL1 classifier could be applied, we analyzed the immunological correlation of B7H4 in different types of cancers using RNA-seq datasets from the TCGA project. We found that $\mathrm{B} 7 \mathrm{H} 4$ expression was negatively correlated with immunomodulators, immune checkpoints and TIICs infiltration in testicular germ cell tumor and $\mathrm{CeCa}$ (Fig. 2A-C). Thus, we chose $\mathrm{CeCa}$ for further analysis due to its relatively high incidence.

\section{B7H4 and PDL1 are mutually exclusive expression in $\mathrm{CeCa}$}

Next, IHC analysis was performed on the CeCa TMA using the antibodies against B7H4 and PDL1. The results showed that B7H4 and PDL1 were highly expressed in
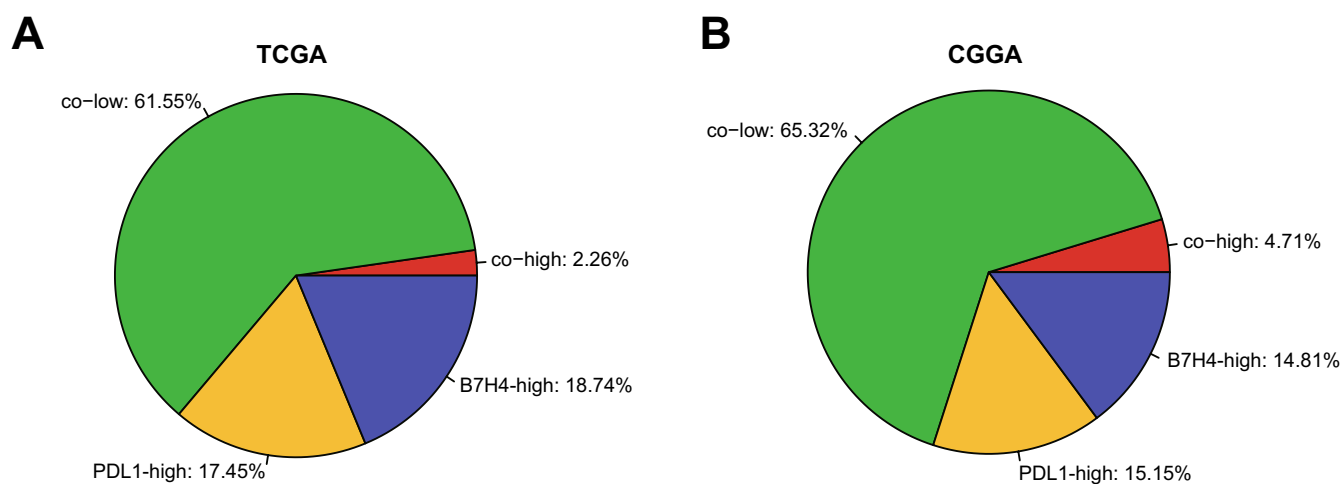

C

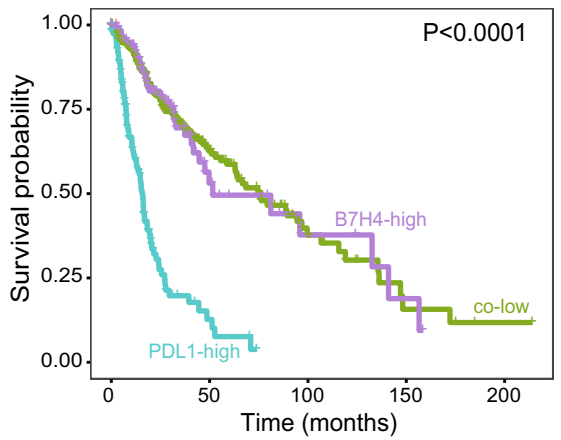

D

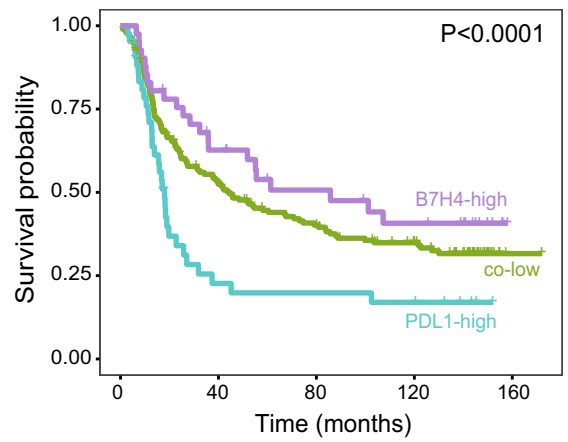

Fig. 1 The prognostic value of the B7H4-PDL1 classifier in glioma. A, B The proportion of B7H4-high, PDL1-high, co-low and co-high subgroups in glioma in TCGA and CGGA dataset. C, D The prognostic value of the B7H4-PDL1 classifier in glioma in TCGA and CGGA datasets 


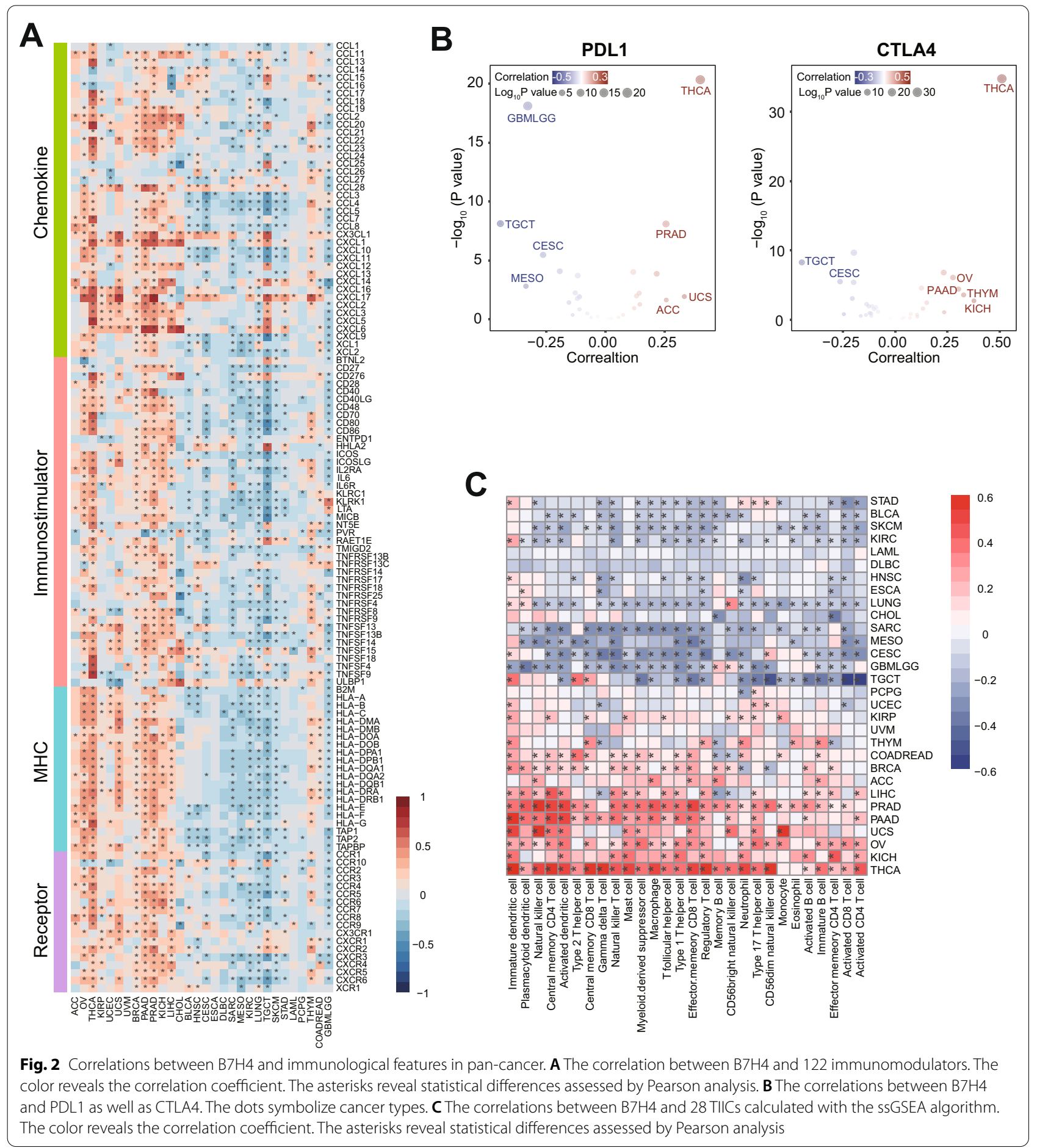

the tumor tissues compared with adjacent tumors, and the positive rates for B7H4 and PDL1 were $19.49 \%$ and $36.44 \%$, respectively (Fig. 3A). Besides, similar to the bioinformatics analysis, $\mathrm{B} 7 \mathrm{H} 4$ expression was negatively correlated with PDL1 (Fig. 3B). T cell inflamed score is developed using IFN- $\gamma$-related mRNA profiles to predict clinical response to PD-1 blockade [20]. In the TCGA dataset, B7H4 expression was negatively while PDL1 expression positively correlated with $\mathrm{T}$ cell inflamed score (Additional file 2: Figure S1A, S1B). Besides, B7H4 was also negatively correlated with most immune checkpoints in addition to PDL1 and CTLA4 (Additional file 2: 
A
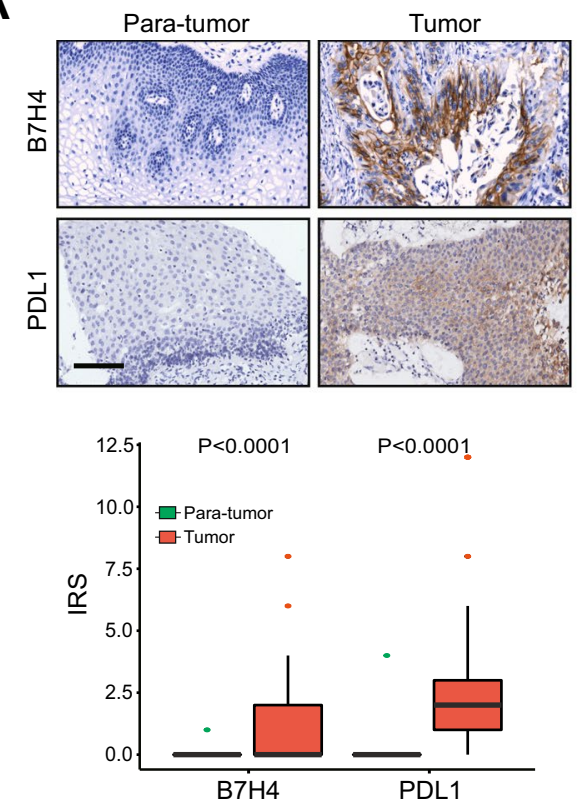

C

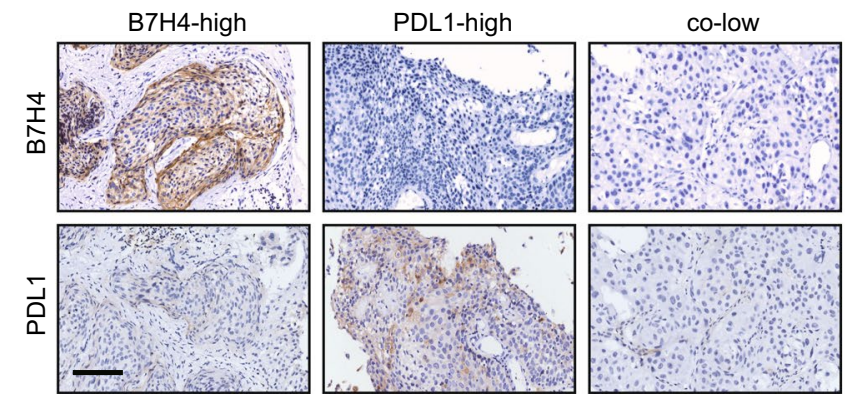

B
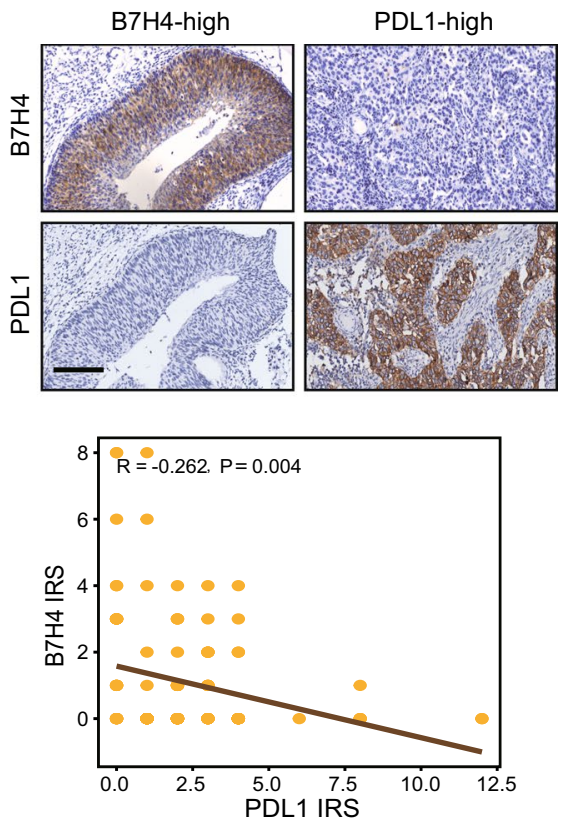

D
E

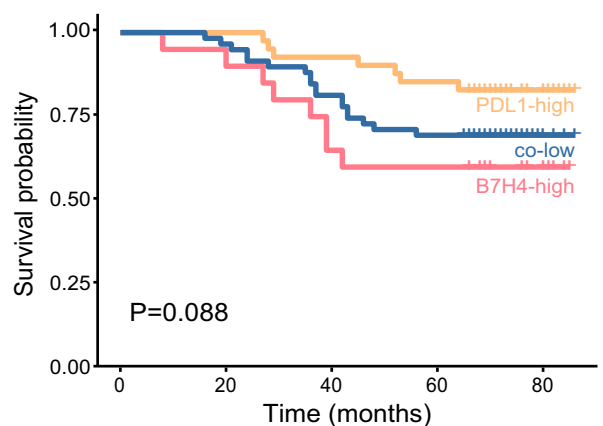

PDL1-high: $33.05 \%$

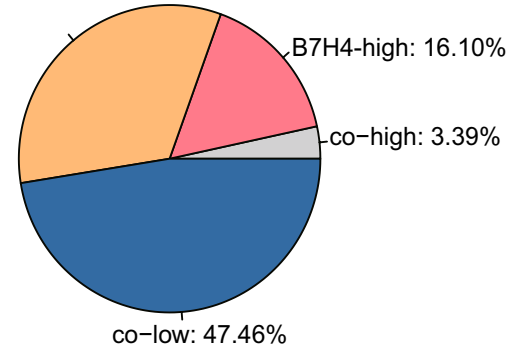

F

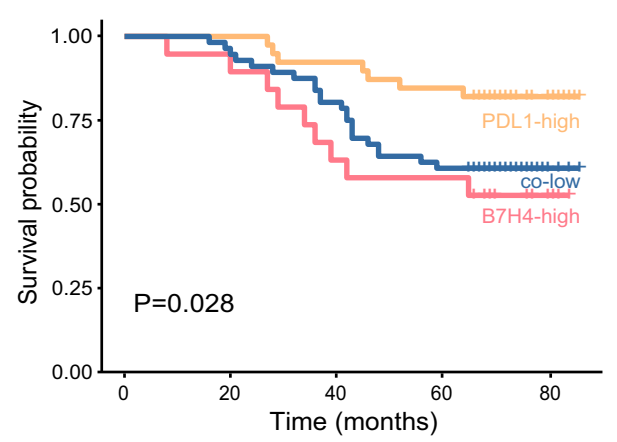

Fig. 3 Expression of B7H4 and PDL1 and prognostic value of the B7H4-PDL1 classifier in CeCa. A Expression levels of B7H4 and PDL1 in CeCa and para-tumor tissues. B The correlation of B7H4 and PDL1 expression CeCa tissues. C Representative images revealing B7H4 and PDL1 expression in different subtypes using anti-B7H4 staining and anti-PDL1 staining. Magnification, $200 \times$, Bar $=100 \mu \mathrm{m}$. D The proportion of B7H4-high, PDL1-high, co-low and co-high subgroups in CeCa. E, F The prognostic value of the B7H4-PDL1 classifier in predicting OS and RFS in CeCa 
Figure $\mathrm{S} 1 \mathrm{C})$. We subsequently divide $\mathrm{CeCa}$ patients into three major subgroups based on B7H4 and PDL1 expression namely B7H4-high, PDL1-high and co-low groups, and the co-high group was excluded from the current analysis due to the limited proportion (Fig. 3C, D). The survival analysis was performed, and the results showed that patients with high $\mathrm{B} 7 \mathrm{H} 4$ exhibited the shortest OS and relapse-free survival (RFS) while those with high PDL1 exhibited a better prognosis (Fig. 3E, F). Taken together, these data support that the B7H4-PDL1 classifier may be suitable in $\mathrm{CeCa}$.

\section{B7H4-high identifies immuno-cold tumors in $\mathrm{CeCa}$}

We next evaluated whether the B7H4-PDL1 classifier was correlated with tumor immunogenicity in CeCa. For the patients in the TCGA dataset, we defined PDL1 as a positive expression with the threshold of top $35 \%$ and B7H4 as a positive expression with the threshold of top $20 \%$ by referring to a positive rate in the IHC cohort. Then, three major subgroups based on B7H4 and PDL1 expression were divided (Additional file 3: Figure S2A). As expected, according to the ESTIMATE algorithm, the B7H4-high group exhibited the lowest stromal score, immune score and ESTIMATE score, but highest tumor purity (Fig. 4A). In addition, most immune cell markers were lowly expressed in the B7H4-high group (Additional file 3: Figure S2B). We also estimated the infiltrating abundance of TIICs using three independent algorithms, and the results also supported that B7H4-high identifies immuno-cold tumors with the lowest TIICs infiltration (Fig. 4B). Besides, activities of most steps in the cycle were revealed to be lowest in the B7H4-high group (Fig. 4C). Moreover, $\mathrm{T}$ cell inflamed score was also lowest in the B7H4-high group (Additional file 2: Figure S2C). We also performed multiplexed QIF using the antibodies against B7H4, PDL1 and CD8 (Fig. 4D). As expected, the B7H4-high group exhibited the lowest CD8 $+\mathrm{T}$ cell levels and the PDL1-high group exhibited the highest CD8 $+\mathrm{T}$ cell levels (Fig. 4E). Collectively, these data support that B7H4-high identifies immuno-cold tumors in CeCa.

\section{B7H4-high identifies highly proliferative tumors and predicts limited therapeutic potions in $\mathrm{CeCa}$}

Subsequently, we evaluated whether the B7H4-PDL1 classifier was correlated with anti-tumor therapies in CeCa. As shown in Fig. 5A, several positively immune-related gene signatures exhibited the lowest scores in the B7H4-high group, the scores of PPARG and $\mathrm{Wnt} / \beta$-catenin networks were highest in the B7H4high group. In addition to negatively regulating antitumor immunity, these pathways were also correlated with high proliferation [21, 22]. Thus, Ki67 was stained by IHC analysis to compare the proliferation of tumors. We found that Ki67 was highest expressed in the B7H4high group (Fig. 5B). Moreover, findings from the Drugbank database revealed a remarkably lowest response to chemotherapy, anti-ERBB (excluding anti-ERBB2 and anti-ERBB4) therapy, antiangiogenic therapy and immunotherapy in the B7H4-high group (Fig. 5C). Besides, IHC analysis validated that EGFR, the main target for anti-ERBB therapy, was lowest expressed in the B7H4high group (Fig. 5D). Moreover, IC50 of anti-cancer drugs in patients from the TCGA database according to the pRRophetic algorithm was estimated. The results showed patients with high B7H4 expression were more sensitive to common anti-cancer drugs (Fig. 5E). To sum up, $\mathrm{B} 7 \mathrm{H} 4$ is an indicator for highly proliferative subtype and limited therapeutic options.

\section{Discussion}

Solid tumors are made up not only of tumor cells, but also of a large number of stromal cells, including immune cells, fibroblasts, etc.[23]. In principle, tumors could be divided into "cold" or "hot" depending on the characteristics of TME. "Cold" tumors are featured with immunosuppressive TME and resistance to either immunotherapy or chemotherapy, but "hot" tumors exhibit higher response rates to these therapies, which are characterized by $\mathrm{T}$ cell infiltration, increased interferon- $\gamma$ (IFN- $\gamma$ ) signaling, activation of inhibitory checkpoints (CTLA4, PDL1, etc.), genomic instability and the activation of major histocompatibility complex class I (MHCI) $[19,24]$. Driving the formation of "hot" tumors is a gradual and complicated process. Extensive efforts have been made to convert cold tumors to hot tumors, but the clinical applications are limited [25]. However, using reliable biomarkers to distinguish "hot" and "cold" tumors is a convenient and feasible strategy.

PDL1 has been identified as an immunosuppressive molecule that negatively regulates cytotoxic immune cells [26]. However, the correlation between PDL1 expression and anti-tumor immunity remains of considerable controversy

\footnotetext{
(See figure on next page.)

Fig. 4 Correlations between the B7H4-PDL1 classifier and immunological features in CeCa. A Differences in Tumor Purity, ESTIMATE Score, Immune Score, and Stromal Score estimating by ESTIMATE algorithm in B7H4-high, PDL1-high and co-low subgroups. ${ }^{*} \mathrm{P}<0.05$; ${ }^{* * P}<0.001 ;{ }^{* * * P}<0.0001$. B Differences in the levels of TIICs calculated using three algorithms in B7H4-high, PDL1-high and co-low subgroups. C Differences in the various steps of the cancer immunity cycle in B7H4-high, PDL1-high and co-low subgroups. Ns: no significant difference; ${ }^{*} P<0.05$; ${ }^{* *} P<0.01$; ${ }^{* * *} P<0.001$; ${ }_{* * * *}>0.0001$. D Representative images revealing B7H4, PDL1 and CD8 expression in different subtypes revealed by multiplexed QIF. Magnification, $\times 200$, Bar $=100 \mu \mathrm{m}$. E Differences in CD8 + T cell infiltration in B7H4-high, PDL1-high and co-low subgroups
} 
A
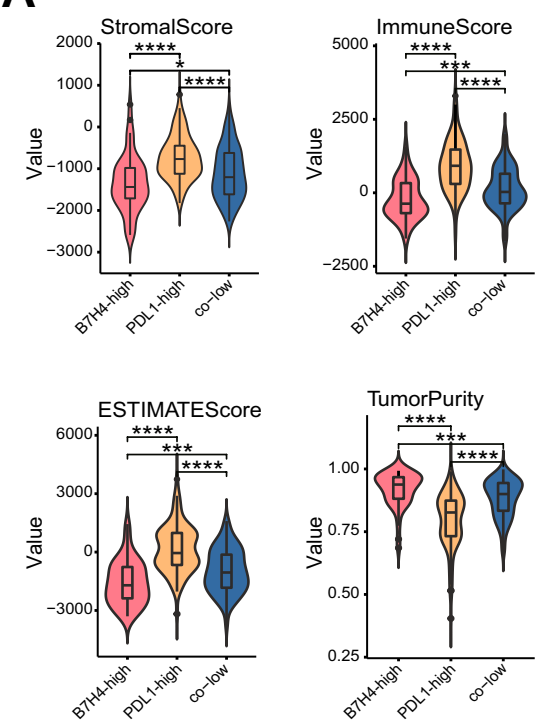

B

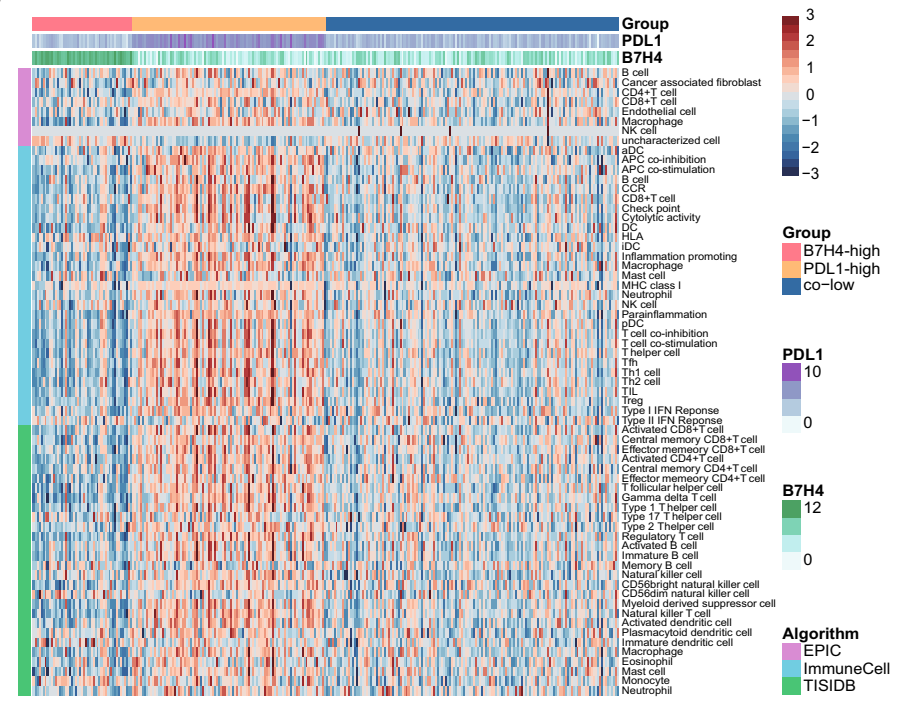

C

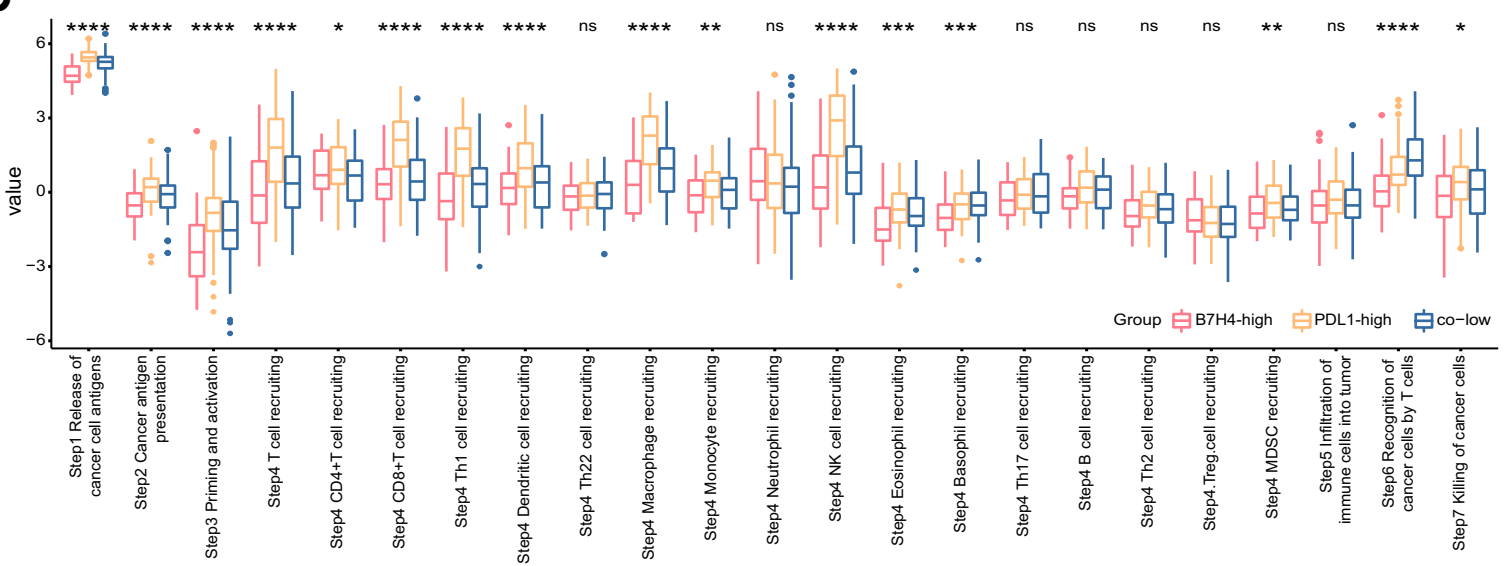

D
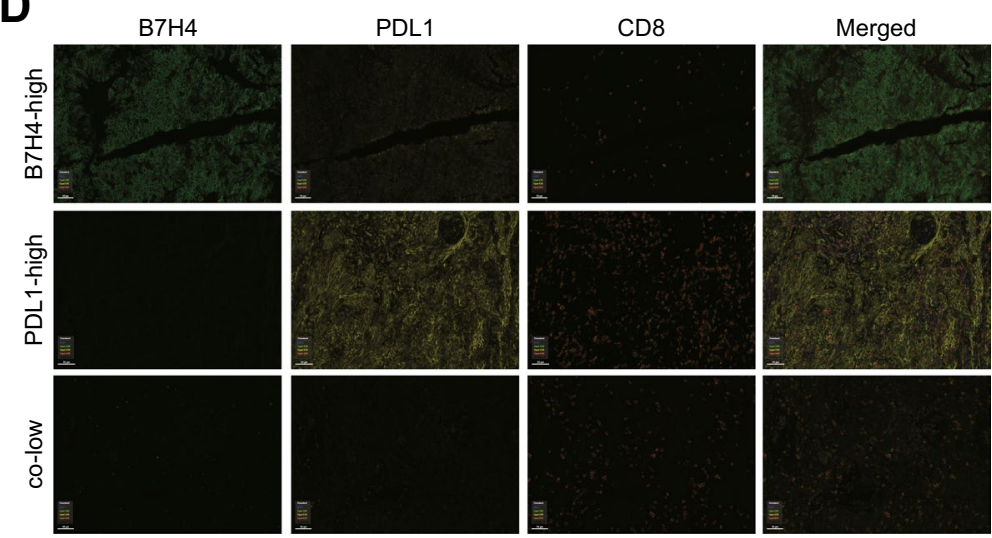

E

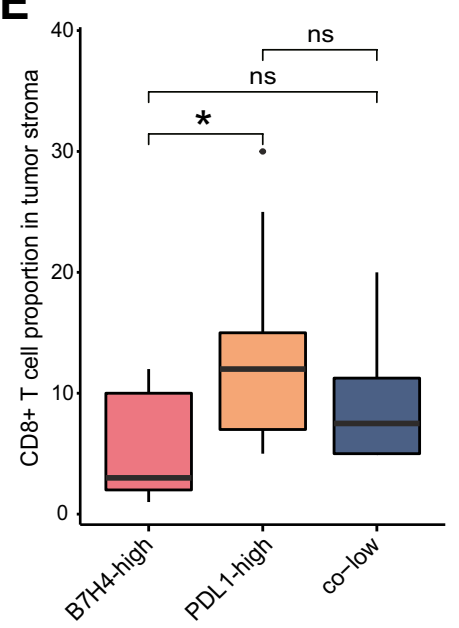

Fig. 4 (See legend on previous page.) 
in human cancers. High PDL1 expression is a striking feature of "hot" tumors and predicts actionable immune activation [27]. Some scholars believe that it is necessary to distinguish constitutive and adaptive PDL1 expression. Constitutive PDL1 has been traditionally considered as a negative co-stimulatory molecule promoted by oncogenic driver mutations [28]. In some cases, PDL1 expression is primarily induced by activated CD8 $+\mathrm{T}$ cells via IFN- $\gamma$ signaling and predicts pre-existing adaptive immune response [29]. Thus, the expression of adaptive PDL1 indicates higher response rates to both immunotherapy and chemotherapy.

B7H4 is another negative co-stimulatory molecule and negatively regulates $\mathrm{T}$ cell activation by inhibiting its activity $[9,10]$. Increasing numbers of research have uncovered that B7H4 was highly expressed in tumor cells and the increased expression predicted poor prognosis in multiple cancers [30-32]. According to the report presented by Chen et al., B7H4 is negatively correlated with PDL1 and identifies immuno-cold tumors in glioma [13]. However, considering that the brain is traditionally an immuneexempt organ as well as the inconsistency between the subtypes demarcated the B7H4-PDL1 classifier and prognosis [14], the application of the B7H4-PDL1 classifier is not a good option in glioma. In this research, we first conducted a pan-cancer analysis of immunological correlation of $\mathrm{B} 7 \mathrm{H} 4$, we found that $\mathrm{B} 7 \mathrm{H} 4$ negatively correlated with immunomodulators, immune checkpoints and TIICs infiltration in $\mathrm{CeCa}$. Further validation suggested $\mathrm{B} 7 \mathrm{H} 4$ and PDL1 were mutually exclusive expression in $\mathrm{CeCa}$. In-depth analysis revealed that the levels of immune infiltration and activation were various in these three groups, and the B7H4-high group exhibited low immune infiltration and activation. In other words, B7H4-high identifies immuno-cold tumors in CeCa. Moreover, the prognosis of these subgroups was consistent with immunogenicity, namely the B7H4-high group exhibited the shortest OS and PFS, which was defined as "cold" tumors.

There is increasing evidence exhibiting that the activation of several oncogenic pathways contributes to the non-inflamed T-cell phenotype and is responsible for the resistance to immunotherapy. Knockdown and pharmacological inhibition of PPARG could notably increase cytokine expression promote the sensitivity to immunotherapy [21]. In Wnt/ $\beta$-catenin-positive melanoma tumors, decreased production of chemokine CCL4 leads to reduced recruitment of BATF3 DCs to the TME [22]. Besides, loss of PTEN activates the PI3K/ AKT pathway, which is associated with an immunocold phenotype in melanoma [33]. In addition, the activation of oncogenic pathways also indicates the highly proliferative capacity of tumor cells. In this research, we found that $\mathrm{B} 7 \mathrm{H} 4$ was positively correlated with the activities of PPARG and Wnt/ $\beta$-catenin pathways and identifies highly proliferative subtypes. Moreover, consistent with the phenotype of "cold" tumors, B7H4-high predicts limited therapeutic potions in $\mathrm{CeCa}$.

In recent years, potential biomarkers to distinguish "hot" and "cold" tumors have been studied extensively. Cai et al. reported that IFITM3 was upregulated in inflamed tumors and could be used as a feasible biomarker [19]. Hu et al. uncovered that SIGLEC15 shaped a non-inflamed tumor microenvironment in bladder cancer [34]. There is no doubt that these studies provide new perspectives to this field. In our research, we revealed the B7H4-PDL1 classifier was essential for clinical assessment to demarcate "hot" and "cold" in $\mathrm{CeCa}$, which may be a useful biomarker for immunotherapy. However, the feasibility of these biomarkers was not tested using large-scale immunotherapy cohorts, which was the main shortcomings in our research and these studies. Thus, we believe that the B7H4-PDL1 classifier should undergo large-scale clinical assessment in immunotherapy cohorts before clinical application. Once its reliability could be confirmed, it will contribute to identifying the advantaged population who may benefit from immunotherapy.

\section{Conclusions}

To sum up, we report that B7H4 is negatively correlated with PDL1 expression and immune cell infiltration. Besides, B7H4-high identifies immuno-cold tumors, highly proliferative tumors and predicts limited therapeutic potions in $\mathrm{CeCa}$. Overall, the B7H4-PDL1 classifier is a convenient and feasible biomarker for the demarcation of tumor immunogenicity in $\mathrm{CeCa}$.

\section{(See figure on next page.)}

Fig. 5 B7H4-high predicts proliferative subtype and limited therapeutic options in CeCa. A Differences in enrichment scores of several therapeutic signatures in B7H4-high, PDL1-high and co-low subgroups. B Differences in Ki67 expression B7H4-high, PDL1-high and co-low subgroups. C Differences in the drug-target genes expression in B7H4-high, PDL1-high and co-low subgroups. D Differences in EGFR expression in B7H4-high, PDL1-high and co-low subgroups. E Differences in IC50 of common anti-cancer drugs in B7H4-high, PDL1-high and co-low subgroups. *P<0.05; ${ }^{* * *} \mathrm{P}<0.001$; ${ }^{* * * *} \mathrm{P}<0.0001$ 


\section{A}

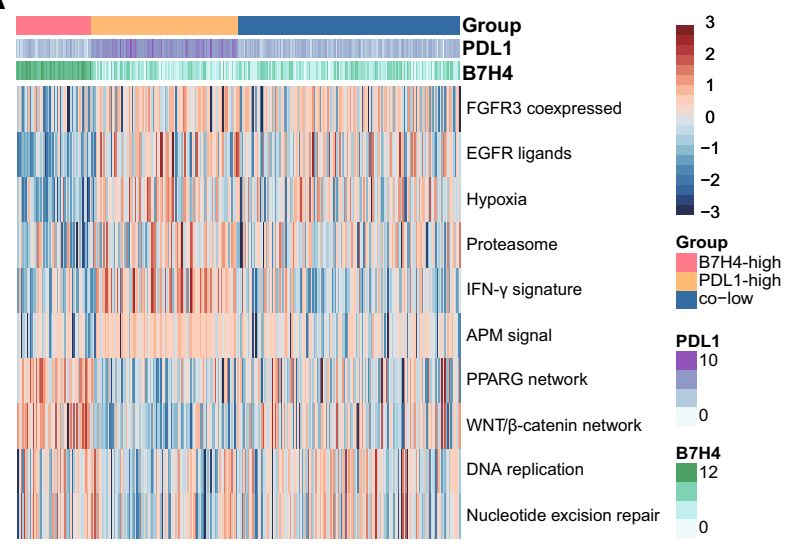

C

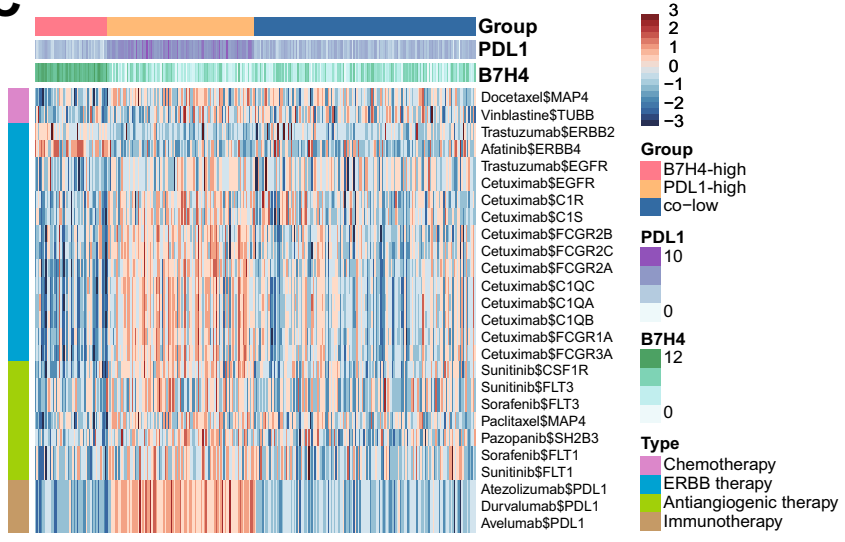

B
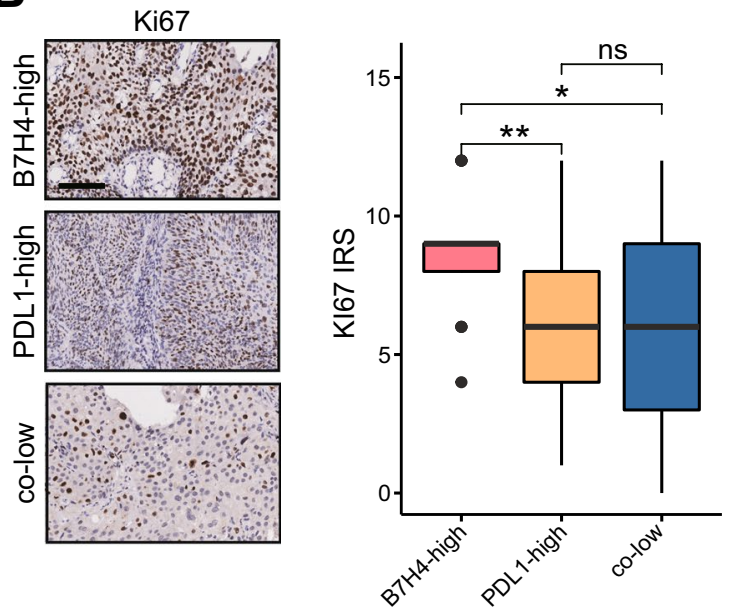

D
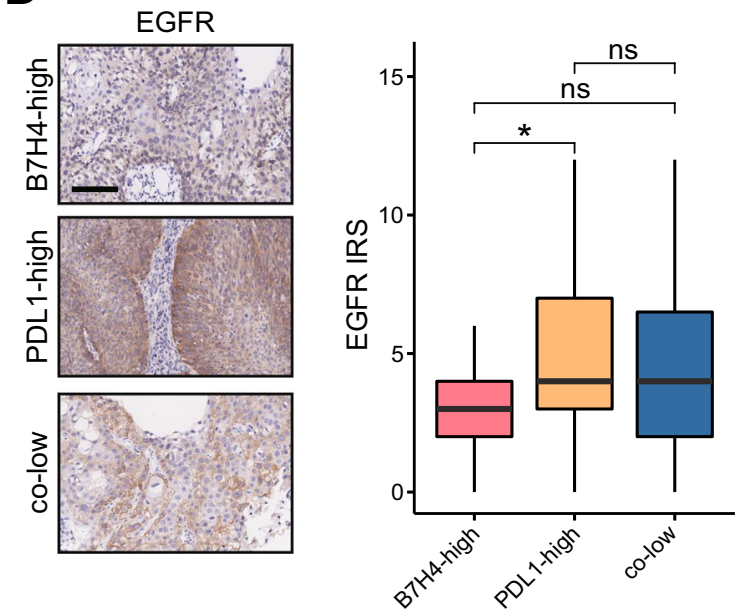

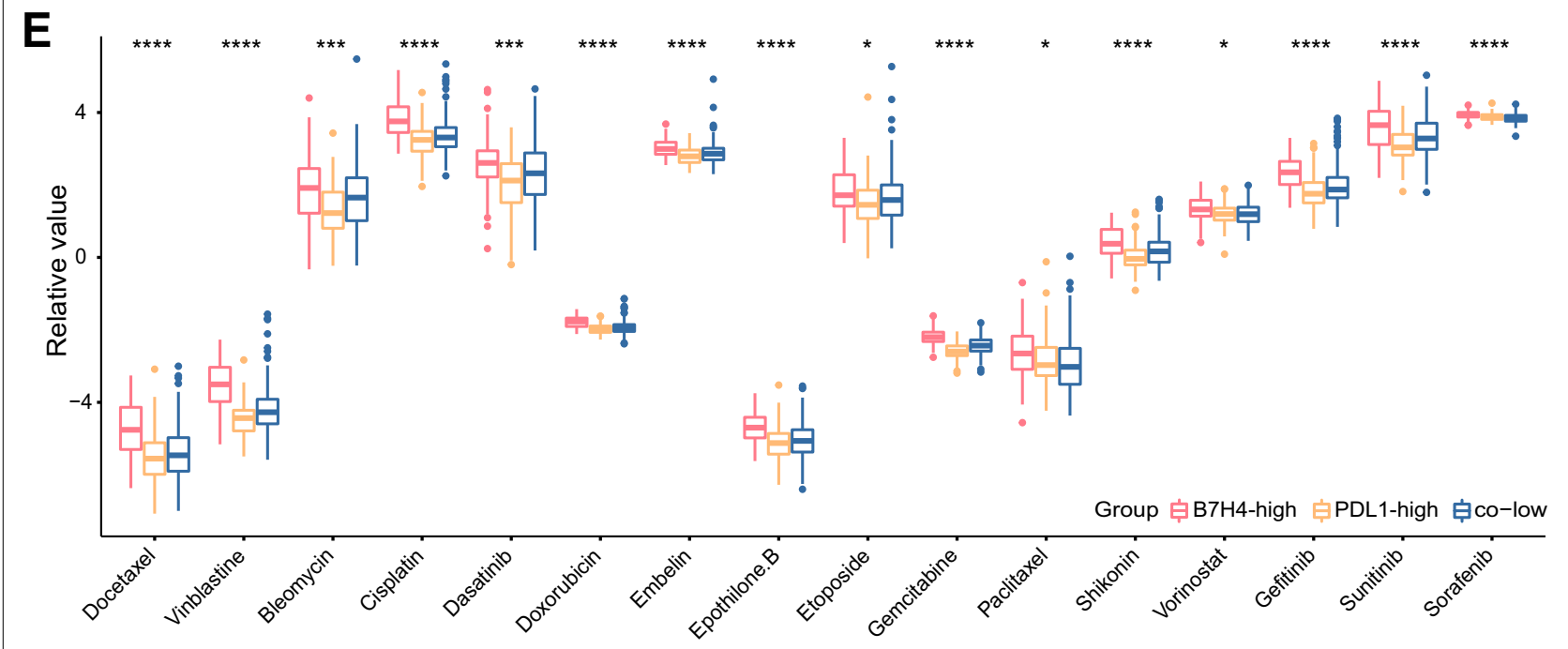

Fig. 5 (See legend on previous page.) 


\begin{abstract}
Abbreviations
ICB: Immune checkpoint blockade; PD1: Programmed cell death 1; PDL1: Programmed death-ligand 1; CeCa: Cervical cancer; TCGA: The Cancer Genome Atlas; CGGA: The Chinese Glioma Genome Atlas. TMA: tissue microarray; QIF: Quantitative immunofluorescence; IHC: Immunohistochemistry; IRS: Immunoreactivity score; OS: Overall survival; RFS: Relapse-free survival; IFN-ү: Interferon- $\gamma$; MHC-I: Major histocompatibility complex class I.
\end{abstract}

\section{Supplementary Information}

The online version contains supplementary material available at https://doi. org/10.1186/s12935-021-02423-8.

Additional file 1: Additional methods; Table S1. Table of abbreviations; Table S2. Detailed information of immunotherapy-related gene signatures.

Additional file 2: Figure S1. Correlations between B7H4 and immunological features in CeCa. (A) The correlation between PDL1 and T cell inflamed score in CeCa. (A) The correlation between B7H4 and T cell inflamed score in $\mathrm{CeC}$. (C) Correlations between B7H4 and common inhibitory immune checkpoints in $\mathrm{CeCa}$. The color reveals the Pearson correlation coefficient.

Additional file 3: Figure S2. Correlations between B7H4-PDL1 classifier and immunological features in $\mathrm{CeC}$ (supplement). (A) The proportion of B7H4-high, PDL1-high, co-low and co-high subgroups in CeCa. (B) Differences in the gene markers of the common TIICs in B7H4-high, PDL1-high and co-low subgroups. (C) Differences in T cell inflamed score in B7H4high, PDL1-high and co-low subgroups.

\section{Acknowledgements}

Not applicable.

\section{Authors' contributions}

YZ and YC conceived and designed the study. LC, JD and ZL performed the assays and bioinformatics analysis. LC and JD prepared the figures and tables. $\mathrm{LC}$ wrote the manuscript. $\mathrm{YZ}$ and $\mathrm{YC}$ revised the manuscript. All authors read and approved the final manuscript.

\section{Funding}

This work was supported by the Major project of Wuxi Science and Technology Bureau (N20201006) to YZ, the Wuxi Double-Hundred Talent Fund Project (BJ2020076) to YZ and Jiangsu Provincial Maternal and Child Health Research Project (F202034) to YZ.

\section{Availability of data and materials}

All data are included in the article, and data are available upon reasonable request.

\section{Declarations}

\section{Ethics approval and consent to participate}

Ethical approval for the use of the TMA was granted by the Clinical Research Ethics Committee in Wuxi Maternal and Child Health Hospital Affiliated to Nanjing Medical University.

\section{Consent for publication}

Not applicable.

\section{Competing interests}

The authors declare no conflict of interest.

\section{Author details}

${ }^{1}$ Department of Oncology, Wuxi Maternal and Child Health Hospital Affiliated to Nanjing Medical University, No. 48 Huaishu Road, Wuxi 214000, China. ${ }^{2}$ Department of Pathology, Wuxi Maternal and Child Health Hospital Affiliated to Nanjing Medical University, Wuxi 214000, China. ${ }^{3}$ Wuxi Clinical Medical College, Nanjing Medical University, Wuxi 214000, China.
Received: 6 September 2021 Accepted: 22 December 2021

Published online: 04 January 2022

\section{References}

1. Hu-Lieskovan S, Ribas A. New combination strategies using programmed cell death 1/programmed cell death ligand 1 checkpoint inhibitors as a backbone. Cancer J. 2017:23(1):10-22

2. Garon EB, Rizvi NA, Hui R, Leighl N, Balmanoukian AS, Eder JP, et al. Pembrolizumab for the treatment of non-small-cell lung cancer. N Engl J Med. 2015;372(21):2018-28.

3. Robert C, Ribas A, Wolchok JD, Hodi FS, Hamid O, Kefford R, et al. Antiprogrammed-death-receptor-1 treatment with pembrolizumab in ipilimumab-refractory advanced melanoma: a randomised dose-comparison cohort of a phase 1 trial. Lancet. 2014;384(9948):1109-17.

4. De Guillebon E, Dardenne A, Saldmann A, Seguier S, Tran T, Paolini L, et al. Beyond the concept of cold and hot tumors for the development of novel predictive biomarkers and the rational design of immunotherapy combination. Int J Cancer. 2020;147(6):1509-18.

5. Mei J, Xu J, Yang X, Gu D, Zhou W, Wang H, et al. A comparability study of natural and deglycosylated PD-L1 levels in lung cancer: evidence from immunohistochemical analysis. Mol Cancer. 2021;20(1):11.

6. Fuchs CS, Doi T, Jang RW, Muro K, Satoh T, Machado M, et al. Safety and efficacy of pembrolizumab monotherapy in patients with previously treated advanced gastric and gastroesophageal junction cancer: phase 2 clinical KEYNOTE-059 Trial. JAMA Oncol. 2018;4(5):e180013.

7. MacGregor HL, Ohashi PS. Molecular pathways: evaluating the potential for B7-H4 as an immunoregulatory target. Clin Cancer Res. 2017;23(12):2934-41.

8. Picarda E, Ohaegbulam KC, Zang X. Molecular pathways: targeting B7-H3 (CD276) for human cancer immunotherapy. Clin Cancer Res. 2016;22(14):3425-31.

9. Prasad DV, Richards S, Mai XM, Dong C. B7S1, a novel B7 family member that negatively regulates T cell activation. Immunity, 2003:18(6):863-73.

10. Sica GL, Choi IH, Zhu G, Tamada K, Wang SD, Tamura H, et al. B7-H4, a molecule of the B7 family, negatively regulates $T$ cell immunity. Immunity. 2003;18(6):849-61.

11. Altan M, Pelekanou V, Schalper KA, Toki M, Gaule P, Syrigos K, et al. B7-H3 expression in NSCLC and Its association with B7-H4, PD-L1 and tumorinfiltrating lymphocytes. Clin Cancer Res. 2017;23(17):5202-9.

12. Gruosso T, Gigoux M, Manem VSK, Bertos N, Zuo D, Perlitch I, et al. Spatially distinct tumor immune microenvironments stratify triple-negative breast cancers. J Clin Invest. 2019:129(4):1785-800.

13. Chen D, Li G, Ji C, Lu Q, Qi Y, Tang C, et al. Enhanced B7-H4 expression in gliomas with low PD-L1 expression identifies super-cold tumors. J Immunother Cancer. 2020;8:1.

14. Sabbagh A, Beccaria K, Ling X, Marisetty A, Ott M, Caruso H, et al. Opening of the blood-brain barrier using low-intensity pulsed ultrasound enhances responses to immunotherapy in preclinical glioma models. Clin Cancer Res. 2021;27:4325-37.

15. Mei J, Yan T, Huang Y, Xia T, Chang F, Shen S, et al. A DAAM1 3'-UTR SNP mutation regulates breast cancer metastasis through affecting miR-208a5p-DAAM1-RhoA axis. Cancer Cell Int. 2019;19:55.

16. Wang Y, Deng J, Wang L, Zhou T, Yang J, Tian Z, et al. Expression and clinical significance of PD-L1, B7-H3, B7-H4 and VISTA in craniopharyngioma. $J$ Immunother Cancer. 2020:8:2.

17. Chen $X$, Chen H, Yao H, Zhao K, Zhang Y, He D, et al. Turning up the heat on non-immunoreactive tumors: pyroptosis influences the tumor immune microenvironment in bladder cancer. Oncogene. 2021;40(45):6381-93.

18. Ribbat-Idel J, Perner S, Kuppler P, Klapper L, Krupar R, Watermann C, et al. Immunologic "Cold" squamous cell carcinomas of the head and neck are associated with an unfavorable prognosis. Front Med. 2021:8:622330.

19. Cai Y, Ji W, Sun C, Xu R, Chen X, Deng Y, et al. Interferon-induced transmembrane protein 3 shapes an inflamed tumor microenvironment and identifies immuno-hot tumors. Front Immunol. 2021;12:704965.

20. Ayers M, Lunceford J, Nebozhyn M, Murphy E, Loboda A, Kaufman DR, et al. IFN-gamma-related mRNA profile predicts clinical response to PD-1 blockade. J Clin Invest. 2017;127(8):2930-40. 
21. Korpal M, Puyang X, Jeremy WuZ, Seiler R, Furman C, Oo HZ, et al. Evasion of immunosurveillance by genomic alterations of PPARgamma/RXRalpha in bladder cancer. Nat Commun. 2017:8(1):103.

22. Spranger S, Bao R, Gajewski TF. Melanoma-intrinsic beta-catenin signalling prevents anti-tumour immunity. Nature. 2015;523(7559):231-5.

23. Binnewies M, Roberts EW, Kersten $K$, Chan V, Fearon DF, Merad M, et al. Understanding the tumor immune microenvironment (TIME) for effective therapy. Nat Med. 2018;24(5):541-50.

24. Gajewski TF. The Next Hurdle in Cancer Immunotherapy: Overcoming the Non-T-Cell-Inflamed Tumor Microenvironment. Semin Oncol. 2015;42(4):663-71.

25. Pelly VS, Moeini A, Roelofsen LM, Bonavita E, Bell CR, Hutton C, et al. Anti-inflammatory drugs remodel the tumor immune environment to enhance immune checkpoint blockade efficacy. Cancer Discov. 2021;11(10):2602-19.

26. Blank C, Gajewski TF, Mackensen A. Interaction of PD-L1 on tumor cells with PD-1 on tumor-specific T cells as a mechanism of immune evasion: implications for tumor immunotherapy. Cancer Immunol Immunother. 2005;54(4):307-14.

27. Hegde PS, Karanikas V, Evers S. The where, the when, and the how of immune monitoring for cancer immunotherapies in the era of checkpoint inhibition. Clin Cancer Res. 2016;22(8):1865-74.

28. Nishino M, Ramaiya NH, Hatabu H, Hodi FS. Monitoring immune-checkpoint blockade: response evaluation and biomarker development. Nat Rev Clin Oncol. 2017;14(11):655-68.

29. Li YM, Yu JM, Liu ZY, Yang HJ, Tang J, Chen ZN. Programmed death ligand 1 indicates pre-existing adaptive immune response by tumor-infiltrating CD8(+) T cells in non-small cell lung cancer. Int J Mol Sci. 2019;20:20.

30. Niu N, Shen W, Zhong Y, Bast RC Jr, Jazaeri A, Sood AK, et al. Expression of $\mathrm{B} 7-\mathrm{H} 4$ and IDO1 is associated with drug resistance and poor prognosis in high-grade serous ovarian carcinomas. Hum Pathol. 2021:113:20-7.

31. Li H, Piao L, Liu S, Cui Y, Xuan Y. B7-H4 is a potential prognostic biomarker of prostate cancer. Exp Mol Pathol. 2020;114:104406.

32. Wang L, Yang C, Liu XB, Wang L, Kang FB. B7-H4 overexpression contributes to poor prognosis and drug-resistance in triple-negative breast cancer. Cancer Cell Int. 2018;18:100.

33. Peng W, Chen JQ, Liu C, Malu S, Creasy C, Tetzlaff MT, et al. Loss of PTEN promotes resistance to $T$ cell-mediated immunotherapy. Cancer Discov. 2016;6(2):202-16.

34. Hu J, Yu A, Othmane B, Qiu D, Li H, Li C, et al. Siglec15 shapes a noninflamed tumor microenvironment and predicts the molecular subtype in bladder cancer. Theranostics. 2021;11(7):3089-108.

\section{Publisher's Note}

Springer Nature remains neutral with regard to jurisdictional claims in published maps and institutional affiliations.

Ready to submit your research? Choose BMC and benefit from:

- fast, convenient online submission

- thorough peer review by experienced researchers in your field

- rapid publication on acceptance

- support for research data, including large and complex data types

- gold Open Access which fosters wider collaboration and increased citations

- maximum visibility for your research: over $100 \mathrm{M}$ website views per year

At $\mathrm{BMC}$, research is always in progress.

Learn more biomedcentral.com/submissions 